\title{
Analysis and optimization of minor actinides transmutation blankets with regards to neutron and gamma sources
}

\author{
Timothée Kooyman ${ }^{\mathrm{a}, 1}$, Laurent Buiron ${ }^{1}$ and Gérald Rimpault ${ }^{2}$ \\ ${ }^{1} D E N / D E R / S P R C / L E D C$ CEA Cadarache, 13108 Saint Paul lez Durance, France \\ ${ }^{2} D E N / D E R / S P R C / L E P h$ CEA Cadarache, 13108 Saint Paul lez Durance, France
}

\begin{abstract}
Heterogeneous loading of minor actinides in radial blankets is a potential solution to implement minor actinides transmutation in fast reactors. However, to compensate for the lower flux level experienced by the blankets, the fraction of minor actinides to be loaded in the blankets must be increased to maintain acceptable performances. This severely increases the decay heat and neutron source of the blanket assemblies, both before and after irradiation, by more than an order of magnitude in the case of neutron source for instance.

We propose here to implement an optimization methodology of the blankets design with regards to various parameters such as the local spectrum or the mass to be loaded, with the objective of minimizing the final neutron source of the spent assembly while maximizing the transmutation performances of the blankets. In a first stage, an analysis of the various contributors to long and short term neutron and gamma source is carried out while in a second stage, relevant estimators are designed for use in the effective optimization process, which is done in the last step. A comparison with core calculations is finally done for completeness and validation purposes. It is found that the use of a moderated spectrum in the blankets can be beneficial in terms of final neutron and gamma source without impacting minor actinides transmutation performances compared to more energetic spectrum that could be achieved using metallic fuel for instance. It is also confirmed that, if possible, the use of hydrides as moderating material in the blankets is a promising option to limit the total minor actinides inventory in the fuel cycle. If not, it appears that focus should be put upon an increased residence time for the blankets rather than an increase in the acceptable neutron source for handling and reprocessing.
\end{abstract}

\section{Introduction}

In the case of a closed nuclear fuel cycle, minor actinides transmutation is a potential solution to further decrease the radiotoxicity burden of the spent fuel, along with the footprint of the final geological repository, by decreasing the long-term activity and decay heat production of the spent nuclear fuel (Chabert, et al., 2011). This is achieved by removing minor actinides from the waste stream and submitting them to a neutron flux in order to obtain shorter-lived fission products.

This neutron flux can be obtained using various means, such as ADS (Tommasi, Bottollier-Curtet, Massara, Varaine, \& Delpech, 2001) or critical fast reactors.. Only such kind of reactors will be considered in this work as successful implementation of minor actinides transmutation requires closure of the fuel cycle which can only be achieved using such reactors.. When considering critical reactors, two approaches can be distinguished. Minor actinides can either be incorporated in the reactor fuel, the so-called homogeneous approach or loaded in dedicated targets named Minor Actinides Bearing Blankets (MABB) located at the periphery of the reactor core. This last option is called heterogeneous transmutation. A detailed analysis of the advantages and drawbacks of each approach can be found in (NEA, 2012). In the homogeneous approach, the neutron spectrum hardening in the core leads to a negative impact on feedback coefficients and on core transient behavior, which means additional safety measures (power reduction, active systems) must be added. The entire fuel cycle is also "polluted" with minor actinides to some extent. However, once an equilibrium situation is reached, minor actinides production in the core is null. In the heterogeneous transmutation case, the "standard" fuel cycle and the transmutation fuel cycle are completely separated and the impacts on core operations are limited as the minor actinides are located in low flux level zones. However, minor actinides production continues in the core itself, which decreases the total transmutation performances of the whole.

To compensate for the low level of flux experienced at the periphery of the core by the transmutation blankets, it is necessary to increase the minor actinides content in the assemblies in order to maintain acceptable transmutation performances - namely in terms of mass consumed per unit of energy produced, usually expressed in $\mathrm{kg} / \mathrm{TWeh}$. This approach is limited by the subsequent increase in decay heat rate and neutron source of the irradiated blanket due to a higher curium production. This increase

\footnotetext{
${ }^{a}$ Corresponding author: timothee.kooyman@cea.fr
} 
lengthens the required cooling time for the irradiated blankets, thus increasing the total minor actinides inventory in the fuel cycle. Additionally, the higher neutron emission increases the radioprotection requirements for handling and transportation of the blankets.

Depending on the corresponding limit for handling or reprocessing fast reactor spent fuel, either irradiated assembly decay heat or neutron emission can constitute a critical point for reprocessing. Considering the high uncertainty remaining on the effective limitations regarding reprocessing, it is currently uncertain which of this parameter will be dimensioning. Consequently, this paper will focus on the behavior of neutron source and associated dose rate with regards to minor actinides transmutation, decay heat considerations being treated separately.

We consider here an equilibrium situation for americium production and consumption in the fuel cycle, where the entire production of americium in the core is matched by consumption in minor actinides bearing blankets. Curium is discarded as a waste during the reprocessing step. Such a situation is for instance discussed in (Meyer et al, 2013).

In this case, the efficiency of the total transmutation process can be characterized by:

- The efficiency of americium destruction during irradiation, which is a measure of the number of reactor units to be equipped with blankets necessary to transmute the amount of americium produced in the cores.

- The total inventory of americium in the fuel cycle. This inventory depends on the irradiation time, the spent fuel cooling time and the manufacturing time of the new assemblies. The cooling time itself depends on the technological constraints associated with reprocessing. This inventory can be linked to the number of transports of radioactive material across a country, which should be as low as possible.

No explicit technological limit for handling or reprocessing spent fuel can be found as of now, considering that such a limit depends on the technological solutions used for assembly handling and transportation and on the reprocessing scheme available in the future, along with radioprotection considerations. However, it is possible to use the corresponding emission level of a standard fuel assembly as a reference point for comparison purposes and to work on a relative scale. This is detailed in the next part.

It has been shown that minor actinides transmutation performances and corresponding neutron source can be fully parametrized by the neutron spectrum and the amount of americium loaded in the blankets (Kooyman \& Buiron, 2015). Considering the simple parametrization of the problem parameters and outputs, an optimization scheme under constraints was implemented in this work. Such a process is discussed here.

The physics of spent target assembly neutron source will be first characterized and compared to a standard fuel cycle assembly. In a second time, the general principle of an optimization methodology of minor actinides transmutation with regards to the fuel cycle constraints and more specifically to radioprotection constraints will be outlined. This methodology will then be applied and the results compared to complete core calculations.

\section{Spent fuel neutron and gamma emissions analysis}

Typical values for Sodium Fact Reactor (SFR) spent fuel after five years of cooling are given in Table 1. They were calculated using the SFR V2B core design as it can be found in (Sciora, et al., 2011). This core is a $3600 \mathrm{MWth}$ homogeneous sodium fast reactor which was designed by CEA, EDF and Areva. Assembly total residence time is 2050 EFPD with a 5-batch management scheme. The ERANOS code system (Rimpault, 2002) was used for core calculations and the DARWIN code system for depletion calculations. (Tsilanizara, et al., 2000).

Table 1 : Spent fuel assembly characteristics after 5 years of cooling

\begin{tabular}{|r|c|}
\hline Assembly mass $(\mathrm{kg})$ & 163 \\
\hline Decay heat $(\mathrm{kW})$ & 1,33 \\
\hline of which alpha (\%) & 55,4 \\
\hline of which beta (\%) & 23,7 \\
\hline of which gamma (\%) & 20,9 \\
\hline Neutron source $(\mathrm{n} / \mathrm{s})$ & $1,22 \mathrm{E}+09$ \\
\hline Mean gamma energy $(\mathrm{MeV})$ & 0,56 \\
\hline Maximum gamma energy $(\mathrm{MeV})$ & 3,40 \\
\hline
\end{tabular}

The neutron source is dominated at $96 \%$ by spontaneous fission of ${ }^{244} \mathrm{Cm}$. Alpha decay heat is mainly coming from ${ }^{244} \mathrm{Cm}(40.7 \%),{ }^{238} \mathrm{Pu}(37.6 \%)$ and ${ }^{241} \mathrm{Am}(8.9 \%)$. Gamma and beta heating is distributed among various fission products. It can be inferred from this analysis that the addition of minor actinides in the fuel will have an impact on the decay heat and the neutron source by increasing the production of ${ }^{244} \mathrm{Cm}$ and ${ }^{238} \mathrm{Pu}$ and the amount of ${ }^{241} \mathrm{Am}$ in the fuel.

For comparison purposes, the same values are computed for a blanket assembly located in the $13^{\text {th }}$ core ring loaded with $20 \%$ in volume of americium oxide $\left(\mathrm{AmO}_{2}\right)$. The results are shown in Table 2. The americium isotopic vector used here is $75 \%{ }^{241} \mathrm{Am}$ and $25 \%{ }^{243} \mathrm{Am}$. The blanket is irradiated for 4100 EPFD as it is considered in (Varaine, Buiron, Boucher, \& Verrier, 2010). 
Table 2 : Spent transmutation target characteristics after 5 years of cooling

\begin{tabular}{|r|c|}
\hline Assembly mass $(\mathrm{kg})$ & 141 \\
\hline Decay heat $(\mathrm{kW})$ & 8,03 \\
\hline of which alpha (\%) & 97,7 \\
\hline of which beta (\%) & 1,4 \\
\hline of which gamma (\%) & 0,9 \\
\hline Neutron source (n/s) & $1,54 \mathrm{E}+10$ \\
\hline Mean gamma energy (MeV) & 0,17 \\
\hline Maximum gamma energy (MeV) & 3,40 \\
\hline
\end{tabular}

A strong shift towards alpha heating in the target can be observed, due to the limited production of fission products compared to a standard fuel assembly. The total gamma power in the fuel assembly is $0.28 \mathrm{~kW}$ compared to $0.07 \mathrm{~kW}$ for the target. In both cases, the maximum energy for a gamma is due to ${ }^{106} \mathrm{Rh}$ decay, which is a fission product. As such, it can be reasonably assumed that the level of gamma shielding provided by handling devices and transportation casks for spent fuel assemblies is enough for target assemblies. For comparison purposes, the gamma spectrum after five years of cooling for an inner fuel assembly and a blanket assembly loaded with minor actinides is given below in Figure 1.

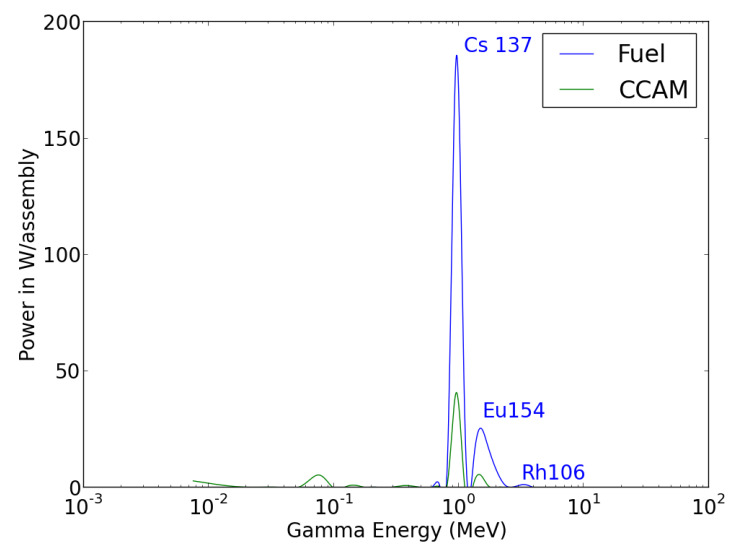

Figure 1 : Comparison of the gamma spectrum for a fuel and CCAM assembly

On the other hand, neutron source increases by a factor twelve between the two cases, which may severely hamper handling and transportation of the irradiated target assemblies if the cooling time is not prohibitively lengthened. Several options exist to make up for this increase, which include design of new transportations casks and handling machines or increased cooling times. However, as the half-live of ${ }^{244} \mathrm{Cm}$ is 18.1 years, a decrease by a factor twelve of the neutron source due to this isotope would require a prohibitive cooling time of 64.5 years. For an irradiation time of 4100 EFPD, this would mean nearly six times as many assemblies cooling down as being irradiated, or 14 tons of americium at various stages of cooling for 2.4 tons being irradiated in a SFR V2b.

Another option which will be investigated in the next part is to locally modify the neutron spectrum near the blankets to limit the production of ${ }^{244} \mathrm{Cm}$ and thus the total neutron source of the assembly. It should be pointed out here that ${ }^{244} \mathrm{Cm}$ production is highly sensitive to the isotopic composition of the americium vector used, as ${ }^{244} \mathrm{Cm}$ is almost only produced through ${ }^{243} \mathrm{Am}(n, \gamma){ }^{244} \mathrm{Cm}$ reactions. This sensitivity will be characterized later on. Neutron spectrum modifications in the blankets have already been discussed, for instance in (De Saint Jean, 1998) or more recently in (Konashi, K.Ikeda, Itoh, Hirai, T.Koyama, \& Kurosaki, 2015).

Considering that the neutron source is dominated by ${ }^{244} \mathrm{Cm}$, the energy spectrum of the neutrons produced in the blankets can be considered constant during cooling and equal to the one of ${ }^{244} \mathrm{Cm}$. This was verified by comparing the neutron spectrum at various cooling times, with mean variations in the neutron spectrum lower than $1.7 \%$ between 5 and 100 years of cooling. Consequently, using dose coefficients taken from (ICRP 119) for anteroposterior neutron exposure of an anthropomorphic phantom and the spectrum shown in Figure 1, it is possible to evaluate the dose coefficient associated with the transmutation blankets neutron source at 317 $\mathrm{pSv} / \mathrm{cm}^{2}$. This value will be used for dose rate calculations in the following part. The contribution of $(\alpha, n)$ reactions is neglected as it is $10^{3}$ lower than the one of spontaneous fission at any given time. The neutron dose rate of a standard fuel assembly after five years of cooling is $0.39 \mathrm{~Sv} / \mathrm{s}$ while the corresponding value of a transmutation target is $4.88 \mathrm{~Sv} / \mathrm{s}$. The standard fuel neutron source (or dose rate) will be considered as the reference level in the next parts of this study.

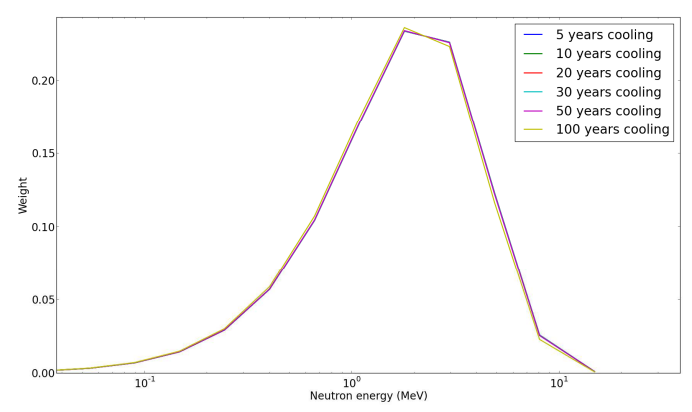

Figure 2 : Comparison of the normalized neutron spectrum at various cooling times

\section{Outline of the optimization approach considered}

We discuss in this part an optimization methodology of minor actinides transmutation blankets with regards to various parameters such as the local neutron spectrum in 
the blankets, the fraction of minor actinides loaded and the maximal acceptable limit for neutron emission at the end of cooling. This approach is based on the consideration that minor actinides transmutation can be characterized considering limited information on the neutron spectrum and the minor actinides loading, as discussed in (Kooyman \& Buiron, 2015).

The r-factor, defined in Eq 1. as the inverse of the difference in neutron lethargy between creation $\left(u_{p}\right)$ and absorption $\left(\mathrm{u}_{\mathrm{d}}\right)$, was used here to parametrize the neutron spectrum. The higher this factor, the more energetic the spectrum is, with $\mathrm{r}$ factor around 0.35 in fuel assemblies and around 0.02-0.05 in hydride-moderated blankets.

$$
r=\frac{1}{u_{p}-u_{d}}
$$

The americium vector used contained $75 \%$ of ${ }^{241} \mathrm{Am}$ and $25 \%$ of ${ }^{243} \mathrm{Am}$. The americium concentration in the target was used as a second parameter and will be denominated $A m$ in the next paragraphs. The maximal acceptable limit for neutron emission at the end of cooling was used as a third parameter and will be denominated $\mathrm{S}_{\mathrm{lim}}$.

The following approach was implemented: an initial calculation with a fixed core configuration with $40 \%$ oxide fuel, $40 \%$ coolant and $20 \%{ }^{56} \mathrm{Fe}$ as structures material was carried out, with $22.1 \%$ of plutonium in the fuel. These values were chosen after considering various SFR designs. It was verified that the spectrum in the core did not influence the spectrum in the blankets. The neutron spectrum is computed using the ECCO cell code with a 33 groups energy mesh and the JEFF 3.1 nuclear data library (NEA/OECD, 2006). Then, this spectrum was used in source-based calculations of a blanket medium with a variable composition in terms of fuel, coolant and moderating material in order to cover as wide as possible a spectrum range. The data used for this approach are given below in Table 3 . The cell calculations were carried out using the ECCO cell code (Rimpault, 2002).

Table 3 : Variation range of used parameters for cell calculations

\begin{tabular}{|c|c|}
\hline Fuel type & $\begin{array}{c}\text { Oxide, Nitride, Carbide, } \\
\text { Metal }(10 \mathrm{w} \% \mathrm{Zr})\end{array}$ \\
\hline Coolant type & $\begin{array}{c}\text { Helium, Sodium, Lead- } \\
\text { Bismuth Eutectic }\end{array}$ \\
\hline Moderating material & $\mathrm{MgO}$, Beryllium, $\mathrm{ZrH}_{2}$ \\
\hline $\begin{array}{c}\text { Moderating material } \\
\text { variation range }\end{array}$ & $0-10$ vol\% \\
\hline $\begin{array}{c}\mathrm{AmO}_{2} \text { volume fraction } \\
\text { variation range }\end{array}$ & $5-40$ vol\% \\
\hline
\end{tabular}

The americium bearing blanket medium is depleted for 4000 EPFD using a constant flux approximation with a flux level of $5 \mathrm{e} 14 \mathrm{n} / \mathrm{cm}^{2} / \mathrm{s}$ representative of what can be found in radial blankets of a SFR V2b. As discussed in [13] for instance, this residence time is compatible with fuel and cladding swelling due to the lower neutron flux at the core periphery. For the core mentioned here above, this corresponds to $2375 \mathrm{~kg}$ of Americium loaded in 84 blankets assemblies. In such a configuration, the americium consumption in the blanket is roughly equal to twice the core production, which means only half of a given reactor fleet must loaded with $\mathrm{MABB}$ to achieve closure of the americium fuel cycle.

In the case of heterogeneous transmutation, the constant flux approximation is deemed realistic enough as blankets are exposed to an almost constant flux level from the core. Various quantities of interest are then computed, namely here transmutation rate and neutron source at various time steps. Other quantities can also be computed, such as decay heat or helium production. One thousand calculations were run to obtain a learning base for the construction of artificial neuron networks which are trained to evaluate the transmutation rate and the neutron source at various time steps $(5,10,20,30,50$ and 100 years) with the Am fraction and the r-factor as input data. This was done using the URANIE platform developed by CEA [26]. The transmutation rate was defined as the ratio of the americium mass consumed over the loaded americium and is expressed in \%: $\tau=\frac{\Delta A m}{A m(t=0)} * 100$ during cooling was approximated using the law described in Eq. 2.

$$
S(t)=a \ln (\mathrm{t})+b t+c \sqrt{t}+d
$$

Considering that the neutron spectrum in the blankets is also dependant on the americium fraction loaded into, artifical neural networks (ANNs) were trained to evaluate the various parameters of interest listed above depending on the r-factor and americium concentration in the fuel. An evaluation of the meta-modelling errors was done and is shown in Table 4. The ANNs were used to compute the neutron source levels at the calculated time steps and then the neutron source behaviour was fitted using the calculated points and Equation 2 as a fit function, as this approach was found to yield the most accurate results. 
Table 4 : Evaluation of some meta-modelling errors for transmutation rate and neutron source at 5 and 50 years. The cases annotated (Ann) correspond to artificial neuron networks calculations and the cases annotated (Reg) correspond to logarithmic regression of the neutron source.

\begin{tabular}{|c|c|c|c|c|c|}
\hline & $\begin{array}{c}\text { Transmutation } \\
\text { rate }\end{array}$ & $\begin{array}{c}\text { Neutron } \\
\text { source } \\
\text { after 5 } \\
\text { years } \\
\text { cooling } \\
\text { (Ann) }\end{array}$ & $\begin{array}{c}\text { Neutron } \\
\text { source } \\
\text { after 50 } \\
\text { years } \\
\text { cooling } \\
\text { (Ann) }\end{array}$ & $\begin{array}{c}\text { Neutron } \\
\text { source } \\
\text { after 5 } \\
\text { years } \\
\text { cooling } \\
\text { (Reg) }\end{array}$ & $\begin{array}{c}\text { Neutron } \\
\text { source } \\
\text { after 50 } \\
\text { years } \\
\text { cooling } \\
\text { (Reg) }\end{array}$ \\
\hline Mean error (\%) & $-0,34$ & 0,06 & $-0,22$ & $-0,09$ & 1,88 \\
\hline $\begin{array}{c}\text { Standard deviation } \\
(\%)\end{array}$ & 4,14 & 3,50 & 3,68 & 3,49 & 3,82 \\
\hline
\end{tabular}

Addition of minor actinides to the blankets has an hardening effect on the neutron spectrum by increasing capture rate in the epithermal energy range. Consequently, the r-factor of the spectrum in the blankets also depends on the Am concentration loaded and not all the combinations $(\mathrm{r}, A m)$ are physically achievable. Using the same approach as the one used to build the initial set, the allowable area in the $(\mathrm{r}, A m)$ plane for the algorithm to explore was computed. This area corresponds to realistic cases in terms of loaded mass and spectrum. Using hydrogenated material such as zirconium hydride $\left(\mathrm{ZrH}_{2}\right)$ as moderator highly increases the allowable area as it can be seen in Figure 3. However, this may lead to a safety concern in case of unprotected transients during which dissociation can occur (Terrani, M.Balooch, Wongsawaeng, S.Jaiyen, \& Olander, 2010). For exhaustiveness and when necessary, we will consider in the following two cases : one with $\mathrm{ZrH}_{2}$ use and one without. In the case without, the allowable area is much lower due to the lower moderating power of materials such as $\mathrm{Be}$ or $\mathrm{MgO}$.

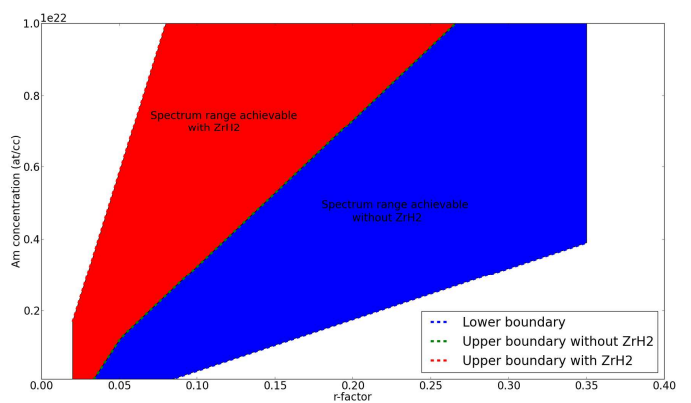

Figure 3 : $(\mathbf{r}, A m)$ diagram. The allowable range without $\mathrm{ZrH}_{2}$ use is located between the two rightmost curves.

Two estimators were used to compare the solutions. The first one is representative of the total heavy nuclides inventory in the blankets. It is calculated based on the following assumptions :

- A minimal cooling time of five years

- A manufacturing time of two years at the end of cooling
We considered that an equilibrium was reached between minor actinides production in the core and consumption in the blankets over the complete fuel cycle. Consequently, if the neutron source after five years of cooling is below $\mathrm{S}_{\mathrm{lim}}$, the inventory estimator is calculated using Eq. 3, with $\mathrm{T}$ being the irradiation time in EFPD:

$$
I(A m, R, T)=\left(1+\frac{7+365}{T}\right) * A m
$$

If not, the cooling time to reach a neutron source equal to $\mathrm{S}_{\mathrm{lim}}$ is approximated by inverting the function used to compute the neutron source and the I estimator is calculated as shown in Eq 4. It can be directly seen that the total inventory depends both on the neutron source of the target and of the considered limit for reprocessing. A maximum cooling time of 100 years was considered here.

$r\left(A m, R, T, S_{l i m}\right)=\left(1+\frac{I_{\text {oooling }}\left(S_{l i m}\right)+Z * 365}{T}\right) * A m$

A second estimator based on the consumption of americium during irradiation is computed using Eq. 5 with $T_{r}$ being the transmutation rate calculated as the percentage of americium having disappeared during irradiation. As the algorithm used minimizes a given objective function, the invert of the americium consumed was used.

$$
C(A m, R)=\frac{1}{A m * T r}
$$

Using the meta-models of the various quantities of interest to compute the value of the estimators within the acceptable $(\mathrm{r}, A m)$ zone, the entire problem is fed to a genetic algorithm for optimization, which is carried with the objective of minimizing I and C, e.g. maximizing the consumption of minor actinides while minimizing the total inventory in the fuel cycle. All of the optimization calculations are carried out using the URANIE code from CEA (Gaudier, 2010) and the python module Scipy (Oliphant, 2007).

It should be mentioned here that an approximation is made here due to technological uncertainties. Indeed, the depletion calculations performed here yield the neutron source per gram of spent fuel. Now, consideration on fuel handling must be computed for an entire assembly, which means this value should be multiplied by the mass of the assembly, which itself depends on the type of fuel or coolant considered. As the information on the geometrical design of the target is not available, this methodology currently only takes into account neutron spectrum effects, regardless of the assembly design. Consequently, the integrated values which are discussed in the next parts are corresponding to a 'reference' target assembly of $141 \mathrm{~kg}$ of heavy metals.

Regarding actual technological feasibility of the designs, the domains shown in Figure 3 were compared with complete core calculations of the SFR V2b described in (Sciora et al, 2011) for various assembly designs using metal and oxide blankets along with various moderating material and were found to be consistent with these calculations. 


\section{Results}

Results are shown in Figure 4, which represents the Pareto front and set for two cases, one where use of zirconium hydride is considered and one where is it not. This two sets corresponds to cases with are optimal in the Pareto-sense, e.g. for which no gain can be achieved for one objective without a loss in another one. In this case, they represent the cases which lead to the minimal cooling time (with regards to neutron source and dose) for a maximum minor actinides consumption. The Pareto front is the set of optimal cases in the initial parameters space while the Pareto zone is the set of optimal cases in the objectives space. It can be seen that, regardless of the use of zirconium hydride, the optimal solutions to the problem corresponds to the least energetic spectrum. However, no cooling time lower than 67 years was observed here, which is prohibitively long and consistent with the values calculated previously using only ${ }^{244} \mathrm{Cm}$ decay information. Consequently, the impact of lowering the neutron source limit for reprocessing was investigated.

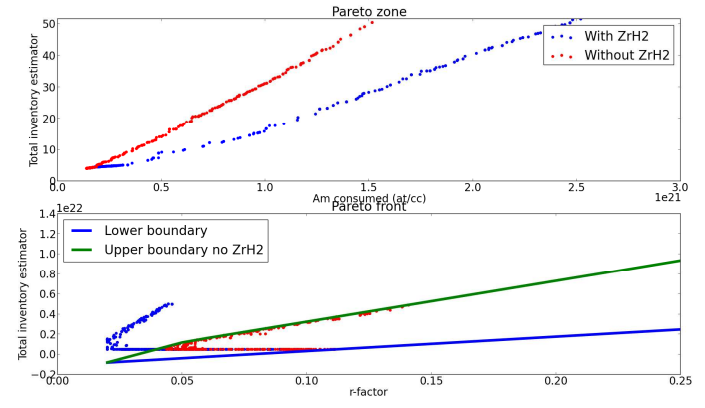

Figure 4 : Pareto front and zone for a considered limit equal to the neutron source of a standard fuel assembly after 5 years cooling $\left(1.2210^{9} \mathrm{n} / \mathrm{s} /\right.$ assembly or $0.39 \mathrm{~Sv} / \mathrm{s} / \mathrm{assembly)}$

The impact of the considered limit on neutron dose rate is shown in Figure 5 . It can be seen that an increase in the allowable limit lead to better solutions in terms of inventories and minor actinides consumption. It can also be seen that increasing the reprocessing limit has an approximately twice bigger effect in the case without $\mathrm{ZrH}_{2}$ compared to the case with $\mathrm{ZrH}_{2}$. Nevertheless, the cases where zirconium hydride is used, even with the reference limit, remains the best option, except for a small subset of cases where the americium consumed is very low. The shape of the Pareto front is also not affected by the raising of the limit considered here.

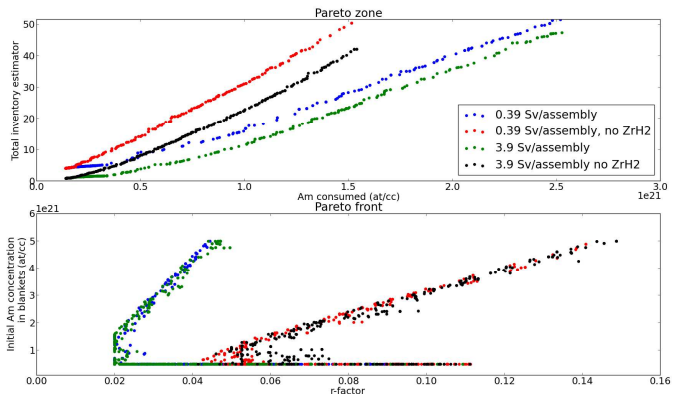

Figure 5 : Impact of the limit considered before reprocessing on the Pareto front and set.

Considering the high contribution of ${ }^{244} \mathrm{Cm}$ to neutron source, the sensitivity of the Pareto set and front to the isotopic vector of americium was also evaluated. Three calculations with varying ${ }^{243} \mathrm{Am}$ fraction were carried out and the results are shown below in Figure 6. For a limit value of $0.39 \mathrm{~Sv} / \mathrm{s}$, it can be seen that the isotopic vector considered has a very limited impact on the Pareto front. Indeed, for most cases, the cooling time required to reach the limit value is beyond 100 years, which is the limiting value considered here. Nevertheless, it can be observed that for high americium fraction, the cases with $10 \% \mathrm{Am}$ 243 exhibit a slightly lower inventory than the one with $40 \%$, which is consistent with the fact that Am 243 is the main precursor or $244 \mathrm{Cm}$.

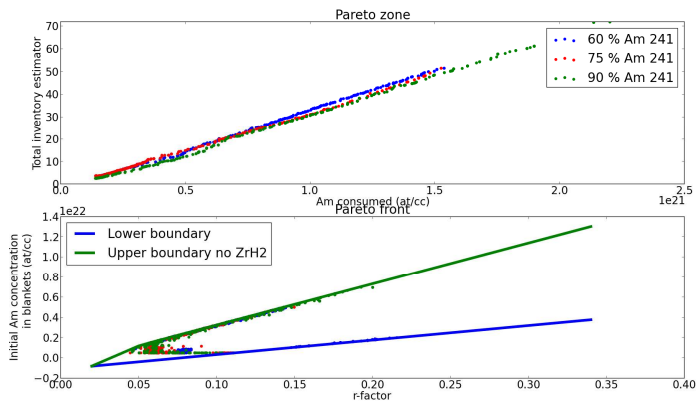

Figure 6 : Impact of the Americium isotopic vector on the Pareto front and set for cases without zirconium hydride. The results are similar when $\mathrm{ZrH}_{2}$ is used. A limiting value of $0.39 \mathrm{~Sv} / \mathrm{s}$ was considered here.

When the limit is increased to $3.9 \mathrm{~Sv}$, as it is done in Figure 7, it can be seen that the impact of the americium vector is increased. As expected, when the ${ }^{243} \mathrm{Am}$ isotopic fraction is increased, the total inventory increases at similar performances. This is explained by the higher production of ${ }^{244} \mathrm{Cm}$ and subsequent increase in the neutron source of the irradiated fuel. Further investigations regarding the impact of the isotopic vector and the possible use of isotopic variations in limiting the total inventory are currently undergoing. This point illustrates the interest is of the optimization approach, as it allows to rapidly explore a wide range of design options. 


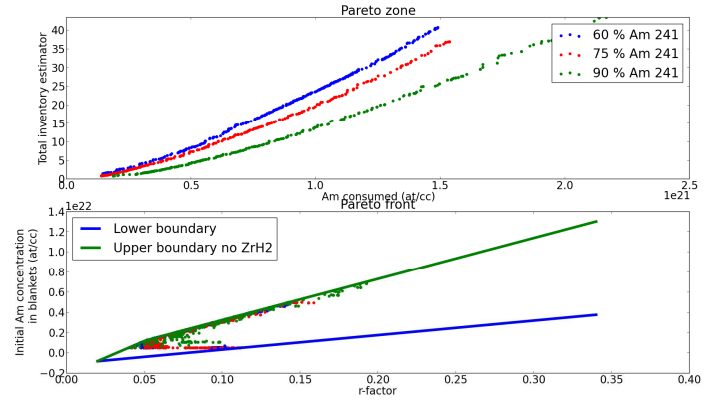

Figure 7 : Impact of the Americium isotopic vector on the Pareto front and set for cases with zirconium hydride. The results are similar when $\mathrm{ZrH2}$ is used. A limiting value of $3.9 \mathrm{~Sv} / \mathrm{s}$ was considered here.

For the irradiation time of 4000 EFPD considered here, it can be concluded that, aside from decreasing the minor actinides loaded in the blankets, the best option to limit the neutron source of the blankets and the cooling time is to use hydrides as moderating material in order to slow down the neutrons at the core periphery. If this option is not available, using moderating material such as beryllium or $\mathrm{MgO}$ appears to be the best option. However, it should be noted that, as it can be seen in Figure 5, the use of hydrides appears as a better solution than an increase in the reprocessing limit for given transmutation performances.

It is also possible to variate the irradiation time to evaluate the impact of this parameter. It can be seen below in Figure 8 that increasing the irradiation time to 6000 EFPD gives identical performances between cases without hydride and cases with hydride irradiated for 4000 EFPD. An increase of the irradiation thus appears as a potential replacement solution to the use of hydrides for neutron slowing-down in the blankets. However, this approach raises additional issues in terms of targets thermo-mechanical behavior at high fluence.

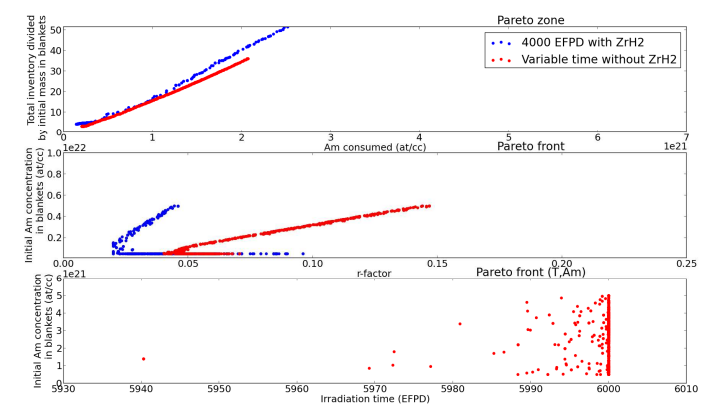

Figure 8 : Comparison of the Pareto front and set for a case with a 4000 EFPD irradiation time and zirconium hydride material vs a variable time without zirconium hydride.

It can also be observed that for the flux level considered here, increasing the irradiation time increases the overall performances. This conclusion may not stay true in the case of higher flux level or homogeneous transmutation due to the so-called 'curium peak' effect (Kooyman \& Buiron, 2015) in which the curium concentration (and the neutron source) increases to a maximum and then decreases after a given fluence.

Finally, it is possible to use $S_{\lim }$ as an input parameter and to run the optimization process using $\mathrm{r}, \mathrm{Am}$ and $\mathrm{S}_{\mathrm{lim}}$ as variables. The comparison between a case without $\mathrm{ZrH} 2$ and a reprocessing limit allowed varying between 0.046 $\mathrm{Sv} / \mathrm{s}$ and $4.6 \mathrm{~Sv} / \mathrm{s}$ and a case with a fixed limit at 0.39 $\mathrm{Sv} / \mathrm{s}$ with $\mathrm{ZrH}_{2}$ is shown in Figure 10. First, it can be seen that the cases with the highest limit are optimal, which was expected. Additionally, it can be seen that for a small part of the americium consumption range (up to $7 \mathrm{e} 20$ at/cc), it is more interesting to increase the reprocessing limit than to use $\mathrm{ZrH} 2$ in terms of total inventory. However, above this threshold, use of $\mathrm{ZrH} 2$ yields the best results. It can be inferred from this and from Figure 5 that raising the maximal allowable neutron source is of interest only for small americium small consumption and that the use of $\mathrm{ZrH} 2$ becomes more interesting above a given consumption value which depends on the reprocessing limit.

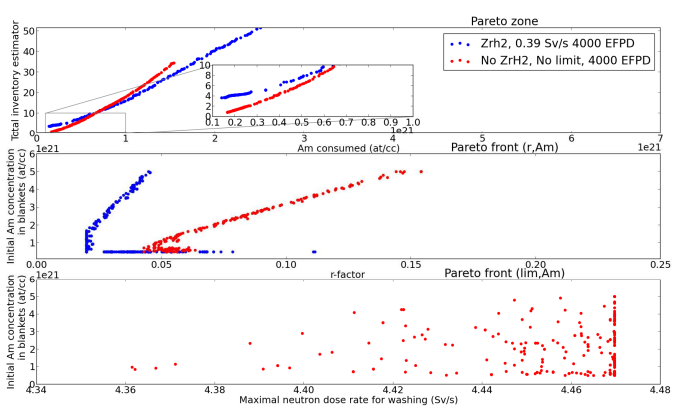

Figure 9 : Comparison of a case with the use of $\mathrm{ZrH} 2$ and a fixed neutron source limit at $0.39 \mathrm{~Sv}$ with a case without $\mathrm{ZrH} 2$ and a variable reprocessing limit

It appears from this analysis, that, apart from using hydrides as moderating material in the blankets, the best option to limit the neutron dose rate at a given time or the cooling time of the blankets is to increase the residence time of the blankets while using an available moderating material such as $\mathrm{MgO}$ or $\mathrm{Be}$ to slow down the neutron. Such an option may not be feasible due to material resistance constraint and especially pin pressurization due to helium release by decaying minor actinides, but the alternative of increasing the allowable limit for reprocessing may also present some technical difficulties due to prohibitive shielding thickness that may be required.

\section{Comparison to core calculations}

Core calculations were carried out to complete and verify the results of the optimization process. The SFR V2B design described in (Sciora, et al., 2011) was also used 
for this purpose. Four cases were compared with similar performances of $6 \mathrm{~kg} / \mathrm{TWeh}$, which corresponds to the one of a "reference" case with $20 \%$ of americium oxide in volume and uranium oxide support matrix:

- The reference case

- A case with oxide and 10 vol\% $\mathrm{ZrH}_{2}$

- A case with oxide and $10 \mathrm{vol} \% \mathrm{MgO}$

- A case with metal fuel (as U10ZrAm with a smear density of $75 \%$ )

The results are shown below in Table 5. It can be seen that the oxide and metal approach are roughly similar, mainly due to the fact that to compensate for the lower transmutation rate in the metal blankets due to the harder neutron spectrum, the amount of initial americium must be raised, thus increasing the overall production of curium. The case with $\mathrm{ZrH}_{2}$ exhibits better performances than the two aforementioned cases, which can be explained by a "shift" towards heavier isotopes which consumes ${ }^{244} \mathrm{Cm}$ to yield ${ }^{245} \mathrm{Cm}\left(+118 \%\right.$ of ${ }^{245} \mathrm{Cm}$ in the moderated case). This shift slightly increases the long term neutron source due to the contribution of ${ }^{246} \mathrm{Cm}$, which increases the cooling time compared to the reference case even though neutron source at 5 years is lower than the reference case. However as the required mass to achieve the same performances is only $71.5 \%$ of the reference mass; the total impact on inventory is limited. The case with $\mathrm{MgO}$ exhibits a similar behavior than the case with $\mathrm{ZrH}_{2}$ but to a lesser extent due to the limited moderating power of $\mathrm{MgO}$. The Am inventory in the blankets necessary to obtain the same performances is slightly reduced while the cooling time is slightly increased due to an increase in the curium production. Consequently, the total americium inventory in the fuel cycle is slightly decreased, but to a lower extent than for the hydride case. These calculations are in good agreement with the results obtained using the methodology. This can also be seen looking at Figure 10, where the cores discussed in Table 5 have been plotted in the Pareto Front diagram from Figure 4. The cores with moderating material, which exhibit the best performances, are lying on the Pareto front, meaning they are optimal in this sense.

Table 5 : Comparison of the performances of oxide, metal and moderated oxide blankets with regards to neutron source

\begin{tabular}{|c|c|c|c|c|}
\hline & Oxide & Metal & $\begin{array}{c}\text { Oxide } \\
+ \\
\mathrm{MgO}\end{array}$ & $\begin{array}{c}\text { Oxide + } \\
\mathrm{ZrH} 2\end{array}$ \\
\hline $\begin{array}{c}\text { Assembly initial heavy } \\
\text { metal mass }\end{array}$ & 141 & 153 & 141 & 141 \\
\hline $\begin{array}{c}\text { Am inventory in } \\
\text { blankets }\end{array}$ & 2375 & 2606 & 2316 & 1699 \\
\hline $\begin{array}{c}\text { 244Cm mass in the } \\
\text { blankets at 5 years (kg) }\end{array}$ & 113,1 & 110,8 & 114,3 & 105,6 \\
\hline $\begin{array}{c}\text { Neutron source at 5 } \\
\text { years (1e10n/s) }\end{array}$ & 1,54 & 1,51 & 1,56 & 1,45 \\
\hline $\begin{array}{c}\text { Cooling time to reach } \\
\text { the level of a standard } \\
\text { fuel assembly (days) }\end{array}$ & 25335 & 24915 & 25455 & 25972 \\
\hline $\begin{array}{c}\text { Estimated americium } \\
\text { inventory (kg) }\end{array}$ & 15476 & 16708 & 15161 & 11342 \\
\hline
\end{tabular}

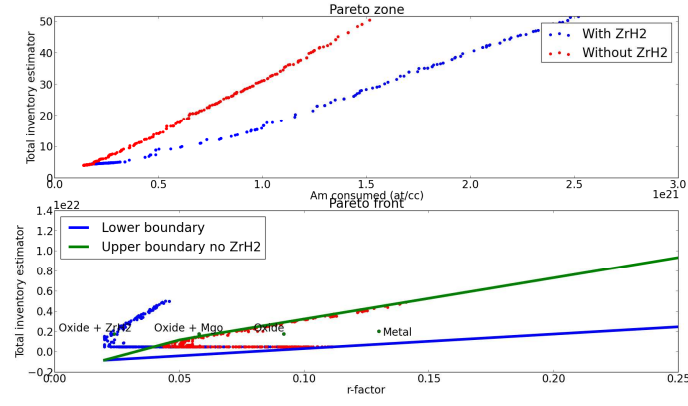

Figure 10 : Pareto Front with position of the cores discussed in Table 5 given

\section{Conclusions}

A new approach to consider transmutation issues related to fuel cycle parameters has been proposed and various heterogeneous transmutation cases have been compared using this methodology. When the spent fuel neutron dose is considered as the limiting factor for reprocessing, it appears that the optimal option in terms of cooling time and minor actinides transmutation performances is to use hydrides as moderating material in the blankets. However, the use of such a material may not be feasible due to potential dissociation issues. In this case, the best option is to use a less-effective moderating material such as beryllium or $\mathrm{MgO}$ and to increase the residence time of the blankets. These findings are consistent with the results of core calculations.

It is also shown here that no optimum can be found for minor actinides transmutation and spent blankets neutron dose rate in terms of neutron spectrum and mass to be loaded. Consequently, other factors such as technological feasibility of the required assembly design will be required to select a unique design option.

Further work will be carried out in the future with regards to the implementation of the optimization methodology, in order to take into account additional parameters linked to the fuel cycle and to core safety. Additionally, extensive analysis of the uncertainties and bias linked to the methodology use will be done.

\section{References}

C. Chabert, C.Coquelet-Pascal, A.Saturnin, G.Mathonniere, B.Boullis, D.Warin et al. : Technical and economic assessment of different options for minor actinides transmutation : the French case. (2011) GLOBAL2011 Proceedings

T.Kooyman, L.Buiron : Sensitivity analysis of minor actinides transmutation to physical and technological parameters. EPJ-N, 1 (15).

NEA Homogeneous versus heterogeneous recycling of transuranics in fast nuclear reactors (2012)

G. Rimpault : The ERANOS code and data system for fast reactor neutronic analyses. PHYSOR Proceedings (2002)

P.Sciora, D.Blanchet, L.Buiron, B.Fontaine, M.Vanier, F.Varaine et al. : Low void effect core design 
applied on 2400 MWth SFR reactor. ICAPP 2011 Proceedings

A.Tsilanizara, C.Diop, B.Nimal, M.Detoc, L.Luneville, M.Chiron : DARWIN: An Evolution Code System for a Large Range. J. of Nuc. Sci \& Tec., Supplement 1, 845-849 (2000)

F.Varaine, L.Buiron, L.Boucher, D.Verrier :Overview on homogeneous and heterogeneous transmutation in a new French SFR : reactor and fuel cycle impact. 11th IEPMT Proceedings (2011).

F.Gaudier : URANIE : The CEA/DEN Uncertainty and Sensitivity platform. Proc. Soc \& Beha. Sci 2 7660-7661 (2010) (2010).

ICRP 119 : Compendium of dose coefficients based on ICRP publication 60. Annals of the ICRP 41 Supplement 1 (2012)

M.Meyer, et al : Scenarios for Minor Actinides Transmutation in the Frame of the French act for waste management Proceedings of FRO13 Conference, Paris. (2013)

K. Konashi et al : Enhancing minor actinides transmutation by irradiation of (MA, $\mathrm{Zr}) \mathrm{Hx}$ in FBR blanket region, Proceedings of GLOBAL2015 Paris (2015).

C. De Saint Jean : Americium once-through of moderated targets in a CAPRA core Seminar Int. CAPRA conf, Karlsruhe (1998) : Scenarios for Minor Actinides Transmutation in the Frame of the French act for waste management Proceedings of FR013 Conference, Paris. (2013)

T.E. Oliphant : Python for scientific computing, Computing in Science \& Engineering 9 (90) (2007).

K. Terrani, M. Balooch, D. Wongsawaeng, S. Jaiyen , D.Olander : The kinetics of hydrogen desorption from and adsorption on zirconium hydride Journal of Nuclear Materials 397 61-68 (2010)

J. Tommasi, H. Bottollier-Curtet, S. Massara, F.Varaine, M.Delpech : Scenarios for waste management involving innovative systems (ADS)

Proceedings of GLOBAL2001 Paris (2001) 